\title{
Métodos de estimativa de porcentagem de cobertura arbórea urbana: um estudo comparativo no Parque Ibirapuera
}

\section{Percent of tree cover estimation methods: a comparative study at Ibirapuera Park}

\author{
Olivatto, Tatiane Ferreira'; Stanganini, Fábio Noel²; Melanda, Edson Augusto³ \\ 1 Universidade Federal de São Carlos (UFSCar). Departamento de Engenharia \\ Civil - Programa de Pós-Graduação em Engenharia Urbana. Rodovia \\ Washington Luís, km 235 - SP-310. São Carlos - São Paulo, CEP 13565-905, Brasil. \\ tatianeolivatto@yahoo.com.br \\ 2 UFSCar, fnsgeo@gmail.com \\ 3 UFSCar, melanda@gmail.com
}

\begin{abstract}
RESUMO
A crescente demanda por planejamento e gestão do ambiente urbano induz à necessidade de indicadores para embasar a tomada de decisões, dentre eles a porcentagem de cobertura arbórea (PTC). Métodos de amostragem aleatória de pontos, como a ferramenta i-Tree Canopy, estimam PTC a partir de imagens do Google Earth. Métodos de sensoriamento remoto, como o Índice de Vegetação por Diferença Normalizada (NDVI) produzem mapas de cobertura arbórea a partir de imagens multiespectrais. Visando comparar estes métodos, ambos foram aplicados no Parque Ibirapuera. Para aplicação do método de amostragem aleatória três condições foram estabelecidas para categorização da amostragem: 500 pontos; desvio padrão inferior à $2 \%$ e 1000 pontos. Para o método de sensoriamento remoto estabeleceram-se três intervalos de NDVI para classificação na categoria "Arbórea", partindo de 0.22 , 0.28 e 0.34 a 1.0. As PTCs obtidas a partir da amostragem aleatória variaram de $60.0 \%$ a $60.4 \%$. No método de sensoriamento remoto, apenas os resultados obtidos considerando NDVI de 0.22 a 1 para "Arbóreo" resultou em PTC de 60.0\%. Desta forma, ambos os métodos se mostraram eficazes na determinação de PTC, sendo necessária a avaliação de aspectos como formato de apresentação desejada e disponibilidade de tempo, recursos e profissionais capacitados.
\end{abstract}

Palavras-chave:, Amostragem Aleatória, i-tree Canopy, Sensoriamento Remoto, Índice de Vegetação por Diferença Normalizada, Porcentagem de Cobertura Vegetal.

\begin{abstract}
The increasing demand for urban environment planning and management induces the need for indicators to support decision making, among them the Percent of Tree Cover (PTC). Random point sampling methods, such as the i-Tree Canopy tool, estimate PTC from Google

${ }^{1}$ OLIVATTO, Tatiane Ferreira; STANGANINI, Fábio Noel; MELANDA, Edson Augusto. Métodos de estimativa de porcentagem de cobertura arbórea urbana: um estudo comparativo no Parque Ibirapuera. In: II SIMPÓSIO NACIONAL DE GESTÃO E ENGENHARIA URBANA: SINGEURB, 2019, São Paulo. Anais... Porto Alegre: ANTAC, 2019.
\end{abstract}


Earth images. Remote sensing methods, such as the Normalized Difference Vegetation Index (NDVI), produce tree cover maps from multispectral images. In order to compare these methods, both were applied at Ibirapuera Park. For random sampling method application, three conditions were established for sampling categorization: 500 points; standard deviation up to $2 \%$ and 1000 points. For remote sensing method three NDVI intervals were established for classification in the "Tree" category, starting from $0.22,0.28$ and 0.34 to 1.0. The PTCs obtained from the random sampling ranged from $60.0 \%$ to $60.4 \%$. In the remote sensing method, only the results obtained considering NDVI from 0.22 to 1 for "Tree" resulted in PTC of $60.0 \%$. Thus, both methods were effective for PTC determining, being necessary the evaluation of aspects as desired presentation format and availability of time, resources and capable professionals.

Keywords: Random Sampling, i-tree Canopy, Remote Sensing, Normalized Difference Vegetation Index, Percent of Tree Cover.

\section{INTRODUÇÃO}

Apesar da preservação dos recursos florestais naturais ser apontada como eixo de sustentação da biosfera, o papel das áreas verdes no ambiente urbano se evidencia nas discussões acerca da qualidade de vida da população urbana. Estas discussões vêm frequentemente acompanhadas de questionamentos sobre a gestão eficiente destas áreas, para que investimentos e gastos com manutenção possam ser justificados e o retorno em serviços possa ser potencializado.

Os serviços proporcionados por estas áreas verdes compreendem os âmbitos ambiental, econômico e social. Destacam-se no aspecto ambiental, serviços como remoção de polventes atmosféricos, amenização de ilhas de calor e interceptação de águas pluviais (PERNA et al., 2017).

Um reflexo da importância destes benefícios é uma crescente tendência internacional pela integração de indicadores ambientais e ferramentas de monitoramento como parte do planejamento e gestão das infraestruturas e dos serviços ecossistêmicos no meio ambiente urbano.

Estimativas de cobertura arbórea são empregadas na elaboração de diversos modelos para compreensão destes serviços ecossistêmicos. Para determinação de porcentagem de cobertura vegetal (PTC, do inglês Percent of Tree Cover) para fins de planejamento e gestão, métodos que dispensam trabalhos exaustivos de campo e que demandam alto investimento são muito utilizados. São eles métodos de amostragem aleatória e métodos de sensoriamento remoto (PARMEHR et al., 2016).

Métodos de amostragem aleatória já eram utilizados para estimativa de cobertura arbórea urbana desde a década de 70. Em 1996, foi apresentada a ferramenta "i-Tree Canopy", desenvolvida pelo Serviço Florestal Americano (USDA Forest Service), que permite estimar a PTC de forma rápida, dispensando obtenção de dados complexos e mão de obra especializada. Desde 2002, a ferramenta é disponibilizada online ${ }^{2}$, sem custos (NOWAK et al., 1996). Metodologias de sensoriamento remoto para a estimativa de PTC se difundiram principalmente a partir da década de 60, paralelamente à disseminação de diversos índices como ferramenta para extração e modelagem de aspectos da vegetação. O Índice de Vegetação por Diferença Normalizada (NDVI, do inglês Normalized Difference Vegetation Index) é um índice amplamente utilizado devido à possibilidade de monitoramento temporal e utilização de imagens de satélites, algumas disponibilizadas livremente, como as do Landsat ${ }^{3}$ (JENSEN, 2009, p. 388).

Considerando a emergente demanda por ferramentas que auxiliem a manutenção do meio ambiente urbano e reconhecendo a relevância dos métodos mencionados, este trabalho focou na comparação entre amostragem aleatória e sensoriamento remoto para estimativa

\footnotetext{
2 Disponível em: https://canopy.itreetools.org/

${ }^{3}$ Disponíveis em: https://earthexplorer.usgs.gov/
} 
de PTC a partir da aplicação destes métodos no Parque Ibirapuera, localizado no município de São Paulo - SP.

\section{METODOLOGIA}

Visando a possibilidade de comparação entre os procedimentos metódicos propostos para estimativa de PTC, ambos foram desenvolvidos na mesma área de estudo, o Parque Ibirapuera, parque urbano do município de São Paulo - SP, de aproximadamente 135 hectares.

\subsection{Amostragem Aleatória}

O método de amostragem aleatória desenvolvido neste estudo utilizou a ferramenta "i-Tree Canopy". A partir da delimitação da área de estudo e com base na diferenciação visual observando-se as imagens do Google Earth, a ferramenta gera pontos aleatoriamente e o usuário é responsável por identificá-los em classes pré-definidas.

Para este estudo foram utilizadas as categorias "Arbórea" (A) e "Outras" (O). A precisão dos resultados obtidos depende de três fatores. O primeiro deles refere-se à habilidade humana de classificar corretamente os pontos, portanto, as categorias escolhidas devem permitir ao usuário diferenciá-las apenas com base nas imagens aéreas. O segundo está relacionado à visibilidade da imagem (por exemplo, ausência de nuvens) (USDA FOREST SERVICE, 2011). O terceiro fator refere-se à quantidade de pontos categorizados. De acordo com o documento de notas técnicas disponibilizado pelo USDA Forest Service (2011), quanto maior o número de pontos, maior é a precisão - e menor o desvio padrão. São sugeridos pelo desenvolvedor de 500 a 1000 pontos. O desvio padrão (SE) calculado por:

$\mathrm{SE}=\sqrt{\frac{p(1-p)}{N}} ;$ sendo $p=\frac{n}{N} ; \mathrm{n}=$ número de pontos de uma categoria; $\mathrm{N}=$ número total de pontos Parmehr et al. (2016) estimou o SE apropriado a partir da relação entre o número de pontos e o valor de PTC (vide Figura 1).

Figura 1 - SE estimado variando com PTC(\%) e N

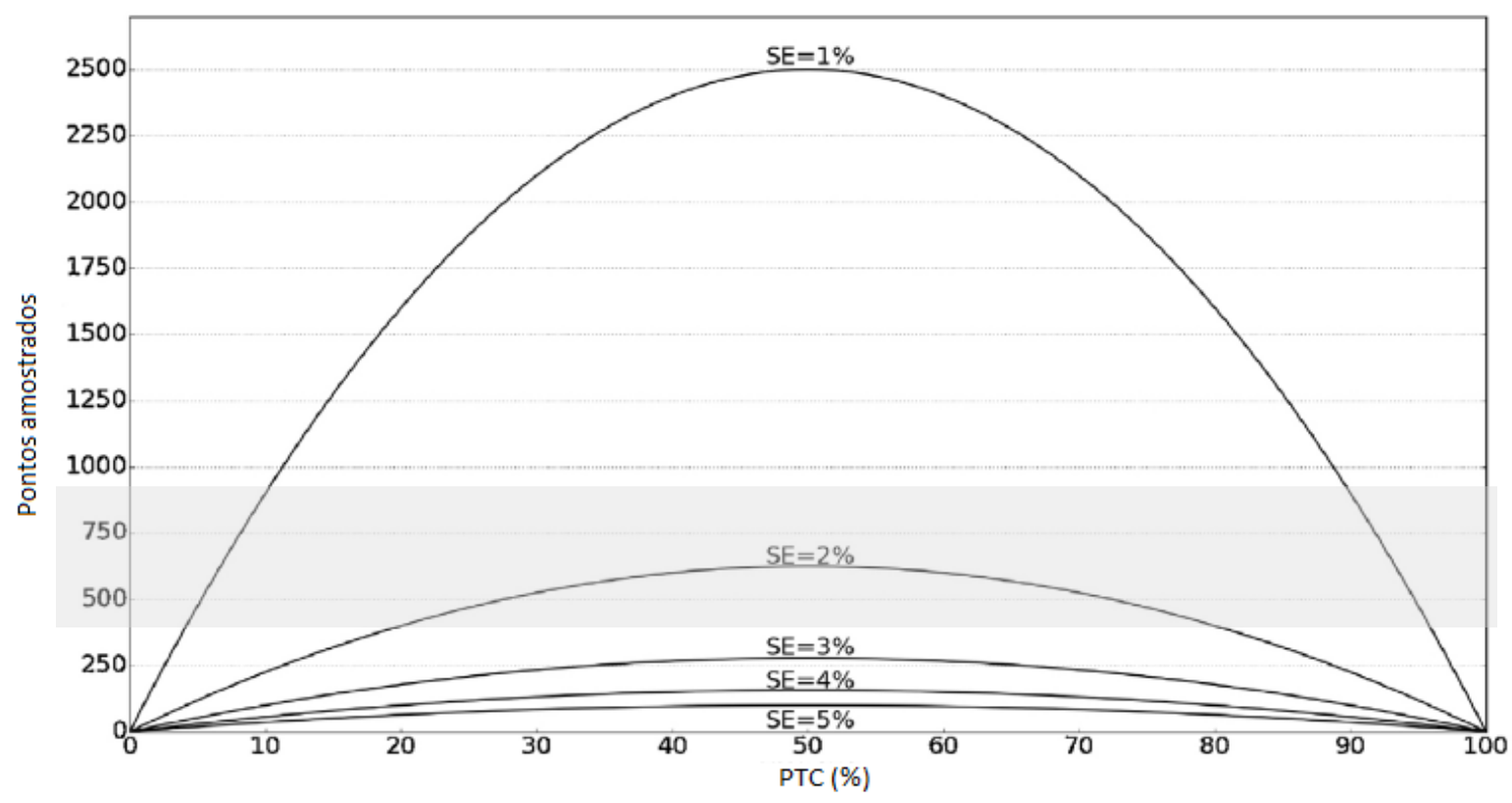

Intervalo de pontos a serem amostrados sugeridos pelo desenvolvedor Fonte: Adaptado de Parmeh (2016) 
Nota-se que para o intervalo de pontos sugeridos pelo desenvolvedor), de 500 a 1000 pontos (destacado em cinza na Figura 1, o SE aproxima-se de 2\%. Com base nestas considerações, o método de amostragem aleatória foi desenvolvido em três condições distintas: $\mathrm{N}=500 ; \mathrm{SE}<$ $2 \%$; $\mathrm{e}=1000$.

\subsection{Sensoriamento Remoto}

O método de sensoriamento remoto para a estimativa de PTC adotado neste estudo foi fundamentado no NDVI. Rouse et al. (1974 apud JENSEN, 2009, p. 386) estabeleceram o NDVI como:

$N D V I=\frac{N I R-R E D}{N I R+R E D} ;$ onde $N I R=$ banda infravermelho próximo e RED= banda vermelho

Para o desenvolvimento deste estudo foram utilizadas as bandas 4 e 5 do Landsat 8 , que equivalem à banda RED e NIR respectivamente, desta forma tem-se:

$\mathrm{NDVI}=\frac{\text { Band } 55-\text { Banda } 4}{\text { Banda } 5+\text { Banda } 4}$

Os valores resultantes variam entre -1 e 1, aproximando-se o maior vigor de vegetação do índice 1. Silva (2014) sugere uma possível correspondência entre valores de NDIV e Vigor de Vegetação (vide Tabela 1).

Tabela 1 - Intervalos NDVI e Vigor de Vegetação

\begin{tabular}{cccc}
\hline Lim. Inferior & Média & Lim. Superior & Vigor de vegetação \\
\cline { 1 - 2 } 0.5 & 0.75 & 1 & Alto/Vegetação Densa \\
0.35 & 0.42 & 0.49 & $\begin{array}{c}\text { Moderado/Vegetação } \\
\text { Baixo/Fragmentos de } \\
\text { Vegetação }\end{array}$ \\
0.22 & 0.28 & 0.34 & $\begin{array}{c}\text { Vaixíssimo/Gramíneas } \\
\text { e/ou Solo Exposto } \\
\text { Ausência/Superfície } \\
\text { Impermeável e/ou } \\
\text { Aquática }\end{array}$ \\
\hline
\end{tabular}

Fonte: Adaptado de Silva (2014) e Jeevalakshmi et al. (2016)

Considerando que parques urbanos se apresentam como fragmentos de vegetação, foram propostas três análises que diferenciam-se pelo intervalo NDVI considerado para categorização (destacado em cinza na Tabela 1). Todos os valores superiores ao limite inferior estabelecido enquadram-se na categoria "Arbórea" (A) e todos os valores abaixo deste limite inferior são considerados "Outras" (O) (vide Tabela 2). O mapa de PTC a partir do NDVI do Parque Ibirapuera foi produzido através do software QGIS ${ }^{4}$, pelo uso da ferramenta de reclassificação.

4 Software livre de sistema de informação geográfica, disponível para download em: https://www.qgis.org/pt_BR/site/ 
Tabela 2 - Intervalos NDVI de Vegetação

\begin{tabular}{|c|c|c|}
\hline \multicolumn{2}{|c|}{ NDVI } & \multirow{2}{*}{ Categoria } \\
\hline Lim. Inferior & Lim. Superior & \\
\hline 0.22 & 1 & Arbórea \\
\hline-1 & 0.22 & Outras \\
\hline \multicolumn{2}{|c|}{ NDVI } & \multirow{2}{*}{ Categoria } \\
\hline Lim. Inferior & Lim. Superior & \\
\hline 0.28 & 1 & Arbórea \\
\hline-1 & 0.28 & Outras \\
\hline \multicolumn{2}{|c|}{ NDVI } & \multirow{2}{*}{ Categoria } \\
\hline Lim. Inferior & Lim. Superior & \\
\hline 0.34 & 1 & Arbórea \\
\hline-1 & 0.34 & Outras \\
\hline
\end{tabular}

Fonte: Os autores

\section{RESULTADOS E DISCUSSÃO}

Para garantir que as diferenças de PCT se referem efetivamente ao método adotado - e não a outros aspectos como alterações na paisagem e desfolhamento sazonal - ambas as análises foram realizadas com base em imagens aéreas datadas de um mês de diferença. A imagem aérea do Google Earth utilizada na amostragem aleatória é de outubro/2018 e a imagem aérea do Landsat 8 utilizada na análise NDVI é de novembro/2018.

\subsection{Amostragem Aleatória}

A categorização dos pontos de amostragem na ferramenta $i$-Tree Canopy foi repetida para 500 pontos, para o desvio padrão máximo de $2 \%$ e para 1000 pontos, resultando respectivamente nos desvios padrões $2.16 \%, 1.99 \%$ e $1.55 \%$ (vide Figura 2).

Cabe esclarecer que o desvio padrão foi limitado à de $2 \%$ pois, como pode ser observado na Figura 1, é indicado para valores de PTC próximos de 60\%, para amostragens entre 500 e 1000 pontos.

Como foi possível observar durante a aplicação das diferentes condições, o acréscimo de pontos amostrados resultou na variação entre 39.6\% e 40\% para a categoria "Outras" e entre $60 \%$ e $60.4 \%$ para cobertura "Arbórea". A vantagem do acréscimo de pontos foi a redução do SE de $2.19 \%$ para $1.55 \%$, aumentando a confiabilidade do método. 
Figura 2 - Porcentagem de ocupação do solo - Parque Ibirapuera

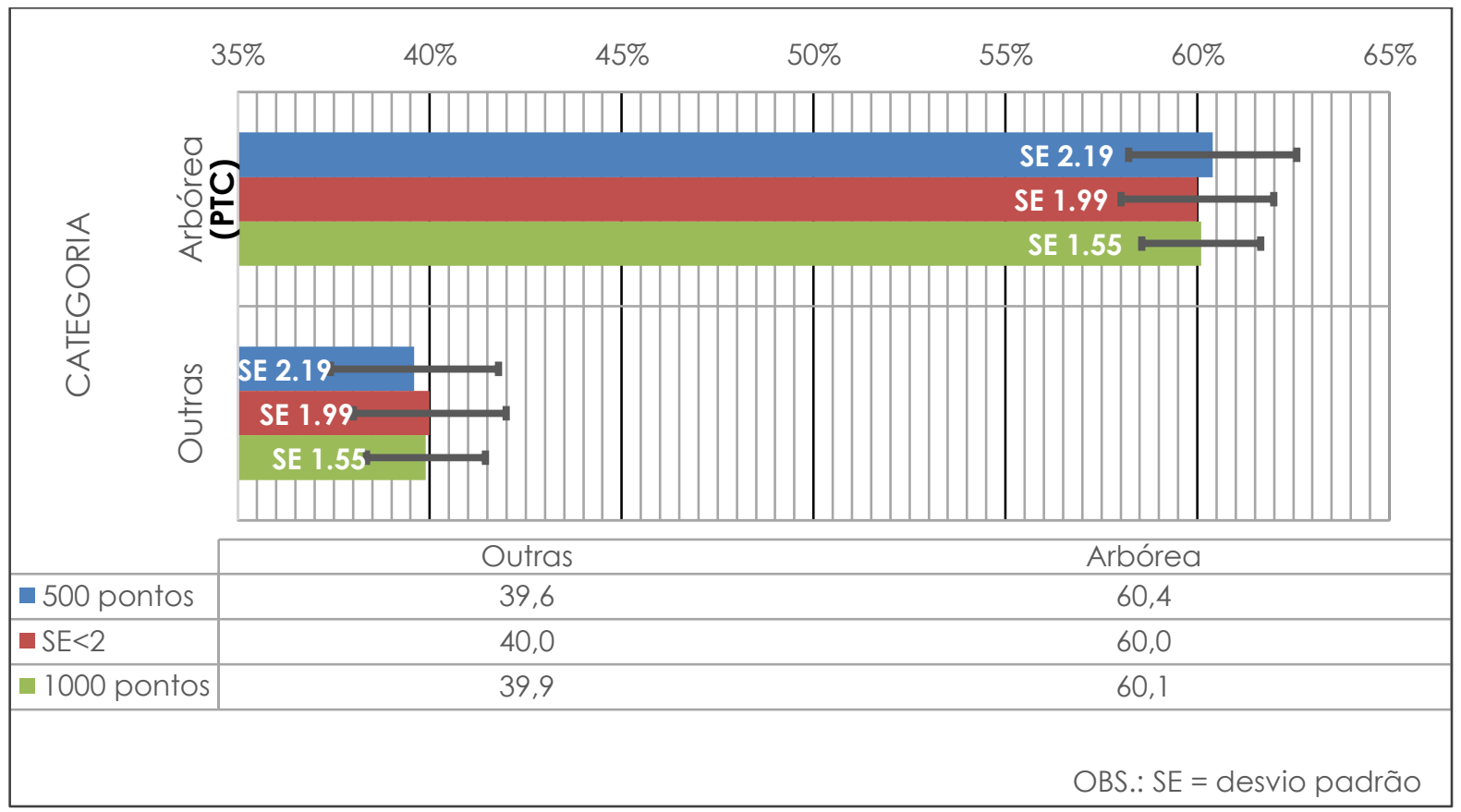

Fonte: Os autores

\subsection{Sensoriamento Remoto}

A imagem aérea e a representação NDVI do Parque Ibirapuera estão dispostos na Figura 3(A) e 3(B), respectivamente.

Figura 3 - Parque Ibirapuera; (A) imagem aérea Digital Globe; (B) resultado NDVI Landsat

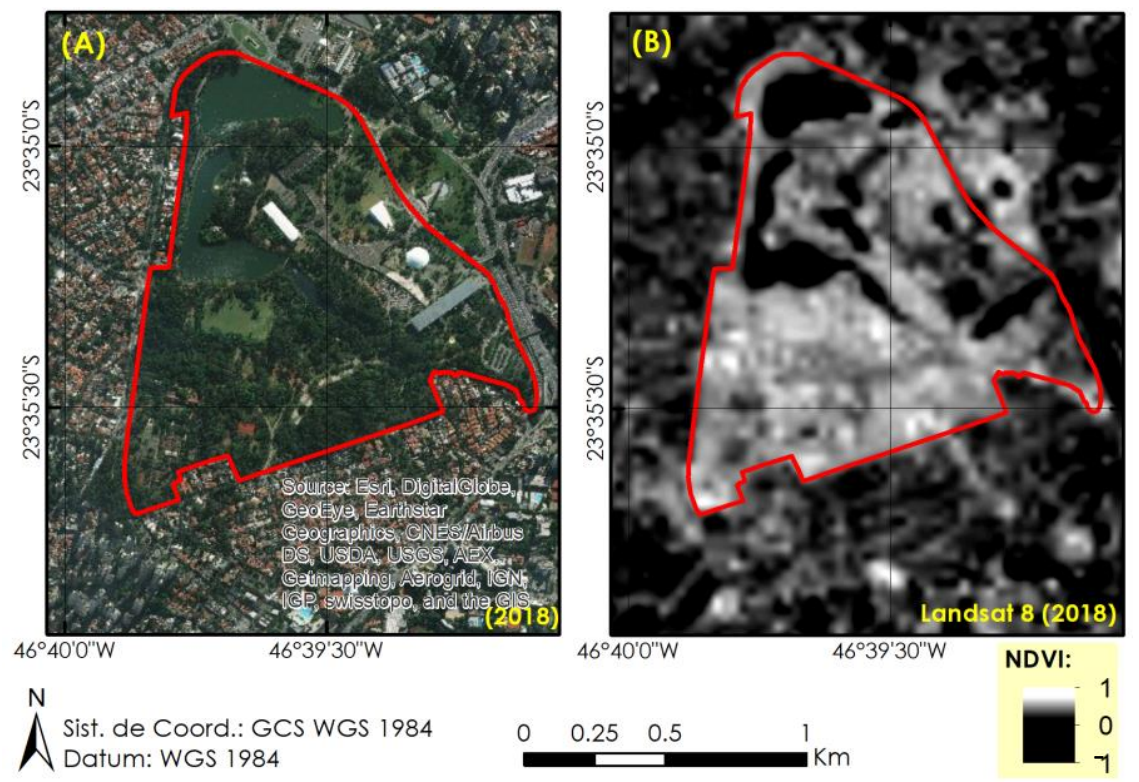

Fonte: Os autores

Na Figura 3(B), as regiões mais escuras correspondem ao índice -1, equivalente a superfícies aquáticas e áreas impermeabilizadas. Comparando-a com a Figura 3(A), percebe-se que a medida que a tonalidade se aproxima do cinza claro, o vigor de vegetação é maior. 


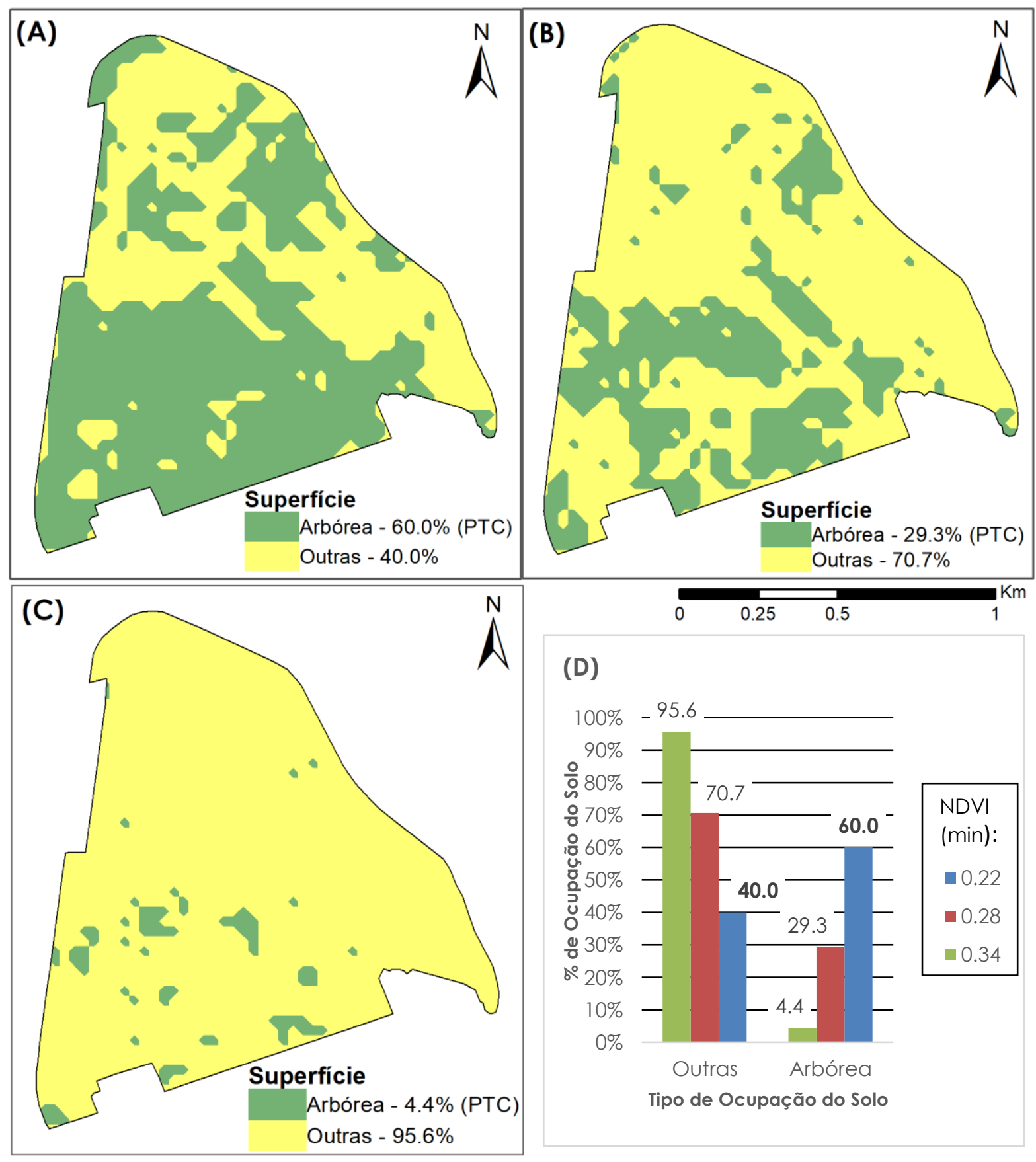

Fonte: Os autores

A análise quantitativa da PTC foi realizada a partir dos valores resultantes da reclassificação dos valores do NDVI de acordo com os intervalos mínimos estabelecidos de $0.22,0.28$ e 0.34 (respectivamente Figura 4(A), 4(B) e 4(C)). Os valores obtidos nestas análises foram agrupados na Figura 4(D) de acordo com as categorias "Arbórea" e "Outras"; já os intervalos mínimos mínimos de NDVI foram representados em diferentes cores.

Quando considerados os limites inferiores de NDVI 0.28 e 0.34 para a categoria "Arbórea", os valores de PTC resultaram respectivamente em $29.3 \%$ e $4.4 \%$. Já quando considerado o limite inferior de NDVI 0.22 para esta categoria, os valores de PTC resultaram em 60.0\%, concordando com os valores de PTC resultantes do método de amostragem aleatória. 


\section{CONCLUSÕES}

A estimativa de PTC é considerada um suporte para o diagnóstico do meio ambiente urbano, sendo os métodos de amostragem aleatória e de sensoriamento remoto utilizados para esta finalidade.

Neste estudo, a análise comparativa entre estes métodos se deu pela utilização da ferramenta i-Tree Canopy, para classificação de pontos amostrados aleatoriamente, e pela reclassificação do NDVI no QGIS, ambos visando o enquadramento da ocupação do solo nas categorias "Arbórea" e "Outras" e, posteriormente, a obtenção da PTC.

Como foi possível observar na aplicação do método de amostragem aleatória, o incremento de 500 pontos resultou numa variação máxima de $0.4 \%$ na PTC. Por outro lado, resultou na redução de $0.64 \%$ no SE. Neste contexto, considerando que a categorização de mais pontos representa aumento significativo no tempo de aquisição de dados, cabe ao usuário refletir sobre a precisão desejada e o tempo disponível.

No método de sensoriamento remoto, a reclassificação do NDVI resultante mostrou-se fator determinante para obtenção da PTC que represente a realidade. Áreas verdes urbanas são caracterizadas por fragmentos de vegetação. A metodologia proposta estabelecendo limites inferiores de NDVI em 0.28 e 034 não identificou todos os fragmentos, mostrando-se inadequados. Já o intervalo de NDVI entre 0.22 e 1 resultou na mesma PTC obtida no método de amostragem aleatória.

Os resultados obtidos permitem realizar algumas avaliações referentes à escolha do método que melhor se adeque às necessidades do usuário.

Diferentemente do método de amostragem aleatória, o método de sensoriamento remoto resulta em um mapa temático, não limitando-se apenas aos valores de PTC. Em contrapartida, necessita de profissionais capacitados para processamento dos dados e produção dos mapas.

A possibilidade de análise temporal da PTC não foi foco deste estudo, contudo cabe ressaltar que esta limita-se principalmente à disponibilidade de imagens. No caso do método de amostragem aleatória, a análise ao longo dos meses e/ou anos limitam-se ao intervalo temporal das imagens disponibilizadas no Google Earth. Neste aspecto, o método de sensoriamento remoto proporciona a possibilidade de escolha de imagens de diversos satélites, os quais possuem variados intervalos temporais. No caso da metodologia NDVI adotada, as bandas NIR e RED são imprescindíveis.

Estudos que auxiliem os tomadores de decisões a planejar e gerir áreas verdes urbanas são relevantes na potencialização dos serviços ecossistêmicos por elas prestados. Em cenários onde disponibilidade de tempo e recursos humanos e financeiros são limitantes, os métodos avaliados neste estudo mostraram-se eficientes na estimativa de PTC.

\section{AGRADECIMENTOS}

Este trabalho foi realizado com apoio da Coordenação de Aperfeiçoamento de Pessoal de Nível Superior - Brasil (CAPES) - Código de Financiamento 001 e do Programa de PósGraduação em Engenharia Urbana (PPGEU) da Universidade Federal de São Carlos (UFSCAR).

\section{REFERÊNCIAS}

JEEVALAKSHMI, D.; REDDY, S. N.; MANIKIAM, B. Land cover classification based on NDVI using LANDSAT8 time series: A case study Tirupati region. In: International Conference on

Communication and Signal Processing (ICCSP), 2016. p. 1332-1335. Disponível em: $<$ ICCSP.2016.7754369>.

JENSEN, J. R. Sensoriamento remoto do ambiente. São José dos Campos: PARENTESE EDITORA, 2009. 
NOWAK, D. J. et al. Measuring and analyzing urban tree cover. Landscape and Urban Planning, v. 36, n. 1, p. 49-57, 1 out. 1996. Disponível em:

<https://www.sciencedirect.com/science/article/pii/S0169204696003246>. Acesso em: 31 mar. 2019.

PARMEHR, E. G. et al. Estimation of urban tree canopy cover using random point sampling and remote sensing methods. Urban Forestry and Urban Greening, v. 20, p. 160-171, 1 dez. 2016. Disponível em:

<https://www.sciencedirect.com/science/article/pii/S1618866716301558>. Acesso em: 31 mar. 2019.

PERNA, A. et al. Implementing and managing urban forests: A much needed conservation strategy to increase ecosystem services and urban wellbeing. Ecological Modelling, v. 360, p. 328-335, 2017.

SILVA, V. C. B. SIG na análise ambiental: Susceptibilidade Erosiva da Bacia Hidrográfica do Córrego Mutuca Minas Gerais. Revista de Geografia, v. 31, n. 2, p. 66-87, 2014.

THOMPSON, S. K. Sampling. 3. ed. New York: John Wiley and Sons, Inc., 2012. Disponível em: <https://onlinelibrary.wiley.com/doi/book/10.1002/9781118162934>.

USDA FOREST SERVICE. i-Tree Canopy Technical Notes. Disponível em:

<https://canopy.itreetools.org/resources/iTree_Canopy_Methodology.pdf >. Acesso em: 31 mar. 2019. 